\title{
The current role of cattle in transmission of hydatidosis in an endemic area of central Iran
}

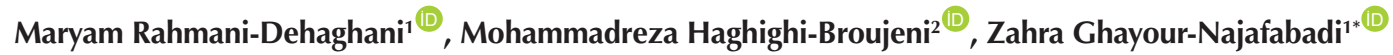 \\ ${ }^{1}$ Department of Parasitology and Mycology, Medical School, Isfahan University of Medical Sciences, Isfahan, Iran \\ ${ }^{2}$ Fasaran Slaughterhouse, Isfahan, Iran
}

*Corresponding Author: Zahra Ghayour-Najafabadi, Tel: 03137929171, 0913329127, Email: z_ghayour@yahoo.com

\begin{abstract}
Background and aims: Hydatidosis is considered as one of the most important zoonotic parasitic diseases, which has a worldwide distribution with endemic regions in many countries including Iran. The disease has public health and economic importance and its effective control requires sufficient information on the patterns of disease transmission. This study aimed to assess the current role of the cattle as the intermediate host in transmission of the disease and maintain the evolution of hydatidosis.

Methods: The study was conducted at Isfahan Fasaran abattoir. From among 129 cattle infected with hydatid cyst, 134 liver and lung cysts were transferred to parasitology laboratory. Data including age, sex, the infected organ, the type of cyst (e.g., fertile, sterile, and calcified), and the severity of infection were evaluated for each of the cattle.

Results: Based on the data, $99(76.7 \%)$ of the cases were females and the remaining $30(23.3 \%)$ cases were males, who were in the age range of 5-10 and 2-5 years, respectively. In addition, 385 cysts were found out of the total 134 examined infected livers and lungs and the number of the cysts in infected organs varied between 1 and 14. A significantly higher infection was detected in older cattle $(P<0.05)$ compared to the younger and female ones. Finally, out of the total of 385 collected cysts, $1 \%, 59.8 \%$, and $39.2 \%$ were fertile, sterile, and calcified cysts, respectively.

Conclusion: In general, the total rate of cyst fertility was estimated at $1 \%$ and it seems that the role of cattle led to a reduction in the transmission of the disease. However, due to the species differences in various areas and the better adaptation of the parasite to some intermediate hosts, further studies including more samples are necessary in different regions.

Keywords: Hydatid cyst; Fertile cyst; Sterile cyst
\end{abstract}

Received: 16 November 2017, Accepted: 29 December 2018, ePublished: 30 August 2019

\section{Introduction}

Hydatid cyst is the larvae stage of the Echinococcus granulosus that is the agent of hydatidosis in intermediate hosts, herbivorous animals, and the humans $(1,2)$. Hydatidosis is one of the most significant parasitic and zoonotic diseases in humans in developing and developed countries (3). Recently, the World Health Organization (WHO) has identified hydatidosis as one of the neglected zoonotic diseases $(4,5)$. Although this disease is easily controlled, it is still regarded as a significant health and medical problem in many parts of the world. Various key factors lead to the survival, emergence, and reemergence of hydatidosis in different parts of the world including the Mediterranean and Iran. Such parameters include the presence of infected dogs with E. granulosus, especially the stray dogs, the easy access of dogs to the viscera of the infected livestock, slaughterhouse inadequate facilities for the disposal of contaminated viscera, uncontrolled trade and exchange of animals among countries, the lack of proper health education, and financial constraints $(5,6)$. According to some studies, the common sheep/dog cycle is described as the cycle mainly involved in human contamination, while a high prevalence of parasites is reported in other intermediate hosts (e.g., cattle, camels, and goat) and may represent the reservoirs of dog infection (7-9). Sheep and camel with $88 \%$ and $70 \%$ fertile cysts are considered as the main intermediate hosts, respectively, and cattle with 19\% fertile cysts are the weakest hosts in the development of E. granulosus in Iran $(7,8)$. Recently, various studies have focused on the role of cattle in $E$. granulosus epidemiology in different parts of Iran. The results show that fertility in cattle hydatid cysts is reduced by $5.3 \%$ and $5.4 \%(10,11)$, indicating that the role of the cows has become weaker in the continuation of the life cycle of the parasite in Iran. In addition, the prevalence of the disease in different geographical areas varies depending on such factors as age, sex, socio-cultural activities, and dog behavior. Similarly, lack of up-to-date information leads to a false interpretation of the real significance of each intermediate host in the development of the parasite. Therefore, to monitor, keep, and control the hydatidosis in an endemic area, identifying the role of each intermediate

(C) 2019 The Author(s); Published by Shahrekord University of Medical Sciences. This is an open-access article distributed under the terms of the Creative Commons Attribution License (http://creativecommons.org/licenses/by/4.0), which permits unrestricted use, distribution, and reproduction in any medium, provided the original work is properly cited. 
host is important for maintaining the E. granulosus cycle. Considering the above-mentioned discussions, the current study sought to determine the status of hydatidosis of the cattle, the rates of fertility/sterility of the hydatid cysts, and the viability of their protoscoleces in order to identify the current role of the cattle in the transmission of the disease in Isfahan.

\section{Materials and Methods}

This cross-sectional study (under the ethical code of IR.MUI.REC.1394.3.906) was performed from January 2016 to May 2016 at Fasaran slaughterhouse in Isfahan. Out of 129 cyst-infected carcasses, 134 liver and lungs containing hydatid cyst were transferred to the parasitology laboratory of the medicine and the characteristics related to age, sex, and the type of the infected organ were recorded for each animal.

After checking the infected organs, the number of cysts (the severity of the infection) was investigated as well. In addition, the size of the diameter of the collected hydatid cysts on each organ was measured and classified into small $(<4)$, moderate (4-7), and large cyst (>7) centimeters.

To identify the viability of the cysts, each one was disinfected with $70 \%$ alcohol and the cyst inside fluid was drained with a 21-gauge nylon syringe, followed by checking for the presence or absence of protoscoleces using a light microscope (40X). Then, the cysts were categorized into fertile and unfertile cases based on the presence or absence of the broad capsule containing protoscoleces in hydatid fluid. To investigate the viability of protoscoleces, a sediment drop containing protoscoleces was observed under the microscope and the viability of protoscoleces was determined by their amoeboid, flame cell movement, and the presence of the calcareous body. Unfertile cysts were divided into sterile and calcified cysts as well. Finally, sterilized hydatid cysts were detected by a smooth inner layer without protoscolex, while calcified cysts were identified by the sound of pebble and sand when splitting.

The collected data were analyzed by SPSS software, version 16 and $P<0.05$ was considered statistically significant in all cases.

\section{Results}

A total of $99(76.7 \%)$ and $30(23.3 \%)$ out of 129 infected carcasses with hydatid cyst were females and males within the age range of $5-10$ and $2-5$ years $(2.1 \pm 0.77)$, respectively. There was a significant relationship $(P<0.05)$ between gender and the severity of infection using the Mann-Whitney test.

The frequency of infected cattle based on the age group and cyst information is described in Table 1 . As shown, a significant relationship $(P<0.05)$ was found between the age group, the infection rate, and the severity of infection, which were higher in the oldest age class. The percentage of sterile, calcified, and fertile hydatid cysts were 59.8\% (230/385), 39.2\% (151/385), and 1\% (4/385), respectively.

Likewise, a total of 385 cysts were collected from 129 infected animals, including 305 (79.2\%) and 80 (20.8\%) cases in 105 livers and 29 lungs, respectively. The mean number of the cysts per animal was $3 \pm 2.34$ and the severity of infection differed from 1 to 14 in the infected organ. Further, the highest numbers of cysts were 14 and 8 per liver and lung. Table 2 represents the numbers of hydatid cysts, which were found in each organ.

Based on the results, most of the cysts had sizes $<4$ $\mathrm{cm}(74.3 \%)$ both in the liver $(80.3 \%)$ and the lungs (51.2\%). The chi-square test failed to show any significant relationship between the age of the infected cattle and the size of the cyst.

The results showed that the majority of the small and calcified cysts were in the liver whereas concerning the status of the cysts, the majority of them were sterile $(238 / 385,61.8 \%)$ both in the liver $(178 / 305,58.3 \%)$ and the lungs $(60 / 80,75 \%)$.

It is noteworthy that unfertile cysts were also observed in the liver and lung containing fertile cysts while no calcified cysts were found in these two organs.

\section{Discussion}

As mentioned earlier, hydatidosis is regarded as one of the most essential zoonotic parasitic diseases with a global distribution within the endemic regions in numerous countries including Iran and has public health and economic significance. The effective control of the disease primarily necessitates sufficient information on the patterns of its transmission in endemic areas. Several studies demonstrated that different parameters such as the prevalence of hydatidosis, the size, location, and the type of cyst (e.g., fertility, sterility, and calcification), relative

Table 1. The frequency and percentage of hydatid-cyst infected cattle in age group and data on hydatid cysts

\begin{tabular}{cccccc}
\hline \multirow{2}{*}{$\begin{array}{c}\text { Age } \\
\text { Category }\end{array}$} & $\begin{array}{c}\text { The Number of } \\
\text { Infected Cattles }\end{array}$ & Number & Mean & Fertile & Calcified \\
\cline { 3 - 6 } & $33(25.5 \%)$ & $79(20.5 \%)$ & 2.4 & $2(2.5 \%)$ & $13(16.5 \%)$ \\
$>4-7$ & $50(38.7 \%)$ & $137(35.6 \%)$ & 2.7 & $2(1.4 \%)$ & $68(49.6 \%)$ \\
$>7$ & $46(35.8 \%)$ & $169(43.9 \%)$ & 3.6 & 0 & $70(41.5 \%)$ \\
Total & $129(100 \%)$ & $385(100 \%)$ & 3 & $4(1 \%)$ & $151(39.2 \%)$ \\
\hline
\end{tabular}


Table 2. The sizes (numbers and percentages) of hydatid cysts recovered from the livers and lungs of the cattle and the viability of hydatid cysts recovered from different organs

\begin{tabular}{|c|c|c|c|c|c|c|}
\hline \multirow{2}{*}{ Organ Localization of Hydatid Cysts } & \multirow{2}{*}{ Cyst Size $(\mathbf{c m})$} & \multicolumn{2}{|c|}{ Data on Hydatid Cysts } & \multicolumn{3}{|c|}{ Data on Hydatid Cysts (Viability) } \\
\hline & & No. & $\%$ & Fertile & Sterile & Calcified \\
\hline \multirow{3}{*}{ Liver (total $n=305$ ) } & $<4$ & 245 & 80.3 & 1 & 106 & 138 \\
\hline & $4-7$ & 45 & 14.7 & 0 & 15 & 30 \\
\hline & $>7$ & 15 & 5 & 2 & 3 & 10 \\
\hline \multirow{3}{*}{ Lung (total $n=80$ ) } & $<4$ & 41 & 51.2 & 0 & 17 & 37 \\
\hline & $4-7$ & 35 & 43.7 & 0 & 2 & 23 \\
\hline & $>7$ & 4 & 5.1 & 1 & 0 & 0 \\
\hline
\end{tabular}

to the geographical location, and the strain of the parasite differ in the cattle as a dog-contaminating reservoir (1215).

Furthermore, hydatidosis is significantly different regarding the host sex in the cattle $(10,14,15)$. The results of the present study revealed that females were more frequently affected than males (with a ratio of 3:1) and were more susceptible to E. granulosus infection. Moreover, the parasite is likely to cause a chronic phase of the disease by creating hormonal imbalances (i.e., the increased estradiol and decreased testosterone levels) and the cyst can stay in the host for a long time (16). Additionally, female cattle are slaughtered in older age due to fertility and thus the rate of their contamination is higher (17).

On the other hand, male cattle are usually slaughtered in the age range of 2-4 years, and are probably less infected accordingly. In the present study, hydatidosis males were found to be about one-third (23.3\%) of the female sex (76.7\%). Similarly, there was a significant difference between the age groups so that the prevalence of infection and the mean severity of infection increased with age. These findings are consistent with the results of other studies (16,18-20). The increase and the severity of the contamination can be due to the increased likelihood of infection or cyst development in older animals (19).

Regarding the fertility of the cysts, the findings represented $1 \%, 59.8 \%$, and $39.2 \%$ for fertility, sterility, and calcification rates, respectively. The variation in the fertility rate of the cysts depends on the geographical location and the type of E. granulosus genotype (21). For example, the fertility rates of the cattle cysts were $26.9 \%$, $27.7 \%$, and $18.4 \%$ in Ethiopian studies $(14,22)$, while the related values were reported $5.3 \%$ and $5.4 \%$ in Iran $(10-11,13)$. However, the fertility rate of the cysts in the current study is almost in conformity with the data obtained by previous studies in Iran, in which cattle are taken into account as the weakest host in the life cycle of E. granulosus $(8,10,11,23,24)$. Therefore, it seems that cattle cannot play a major role in the life cycle of parasites in this district of Iran.

The findings of the present study showed that the lung cysts were large and moderate, while those of the liver were small and calcified, probably allowing the cyst to grow more due to the relatively soft tissue of the lungs (2). In addition, the presence of more calcified cysts in the liver can be due to relatively high levels of reticuloendothelial cells and a high response to liver connective tissue. Further, the higher levels of small calcified cysts are probably attributed to host immunologic responses that may inhibit the growth and development of the parasite (25).

\section{Conclusion}

Overall, the fertility rate of cattle hydatid cysts was low and reported to be $1 \%$ in this study, and thus the role of the cattle appeared to be diminished in the transmission of hydatidosis. However, due to the variation in the strains in different regions and better adaptation of the worm to some hosts in certain places, performing further studies in different regions and with more samples is required to identify the causes of contamination to prove this theory.

\section{Conflict of interests}

None.

\section{Acknowledgments}

This article is part of the master's degree thesis in parasitology (under number 394906) in collaboration with Isfahan University of Medical Sciences. We appreciate the collaboration of the Research Deputy and the Department of Parasitology of Isfahan University of Medical Sciences and Fasaran slaughterhouse.

\section{References}

1. Sadjjadi SM. Present situation of echinococcosis in the Middle East and Arabic North Africa. Parasitol Int. 2006;55 Suppl:S197-202. doi: 10.1016/j.parint.2005.11.030.

2. Smyth JD, Wakelin D. Introduction to animal parasitology. New York: Cambridge University Press; 1994.

3. Carmena D, Sánchez-Serrano LP, Barbero-Martínez I. Echinococcus granulosus infection in Spain. Zoonoses Public Health. 2008;55(3):156-65. doi: 10.1111/j.18632378.2007.01100.x.

4. da Silva AM. Human echinococcosis: a neglected disease. Gastroenterol Res Pract. 2010;2010:583297. doi: $10.1155 / 2010 / 583297$.

5. Eckert J, Conraths FJ, Tackmann K. Echinococcosis: an emerging or re-emerging zoonosis? Int J Parasitol. 2000;30(1213):1283-94.

6. Eckert J, Deplazes P. Biological, epidemiological, and clinical aspects of echinococcosis, a zoonosis of increasing concern. Clin Microbiol Rev. 2004;17(1):107-35. doi: 10.1128/ 
cmr.17.1.107-135.2004.

7. Eslami A, Hosseini SH. Echinococcus granulosus infection of farm dogs of Iran. Parasitol Res. 1998;84(3):205-7.

8. Rokni M. Echinococcosis/hydatidosis in Iran. Iran J Parasitol. 2009;4(2):1-16.

9. Ghasemian O, Hoseini G, Soleimani M, Mahmoudi R, Kaboudari A. The prevalence study of hydatid cyst in domesticated slaughtered animals in industrial abattoirs in Iran. J Bacteriol Mycol Open Access.2018;6(2):96-100.

10. Adinehbeigi K, Radfar MH, Rahmani K. The role of cattle in the epidemiology of Echinococcus granulosus in Kerman area, southeast of Iran. Comp Clin Path. 2013;22(2):233-8. doi: 10.1007/s00580-011-1391-4.

11. Daryani A, Sharif M, Amouei A, Nasrolahei M. Fertility and viability rates of hydatid cysts in slaughtered animals in the Mazandaran province, Northern Iran. Trop Anim Health Prod. 2009;41(8):1701-5. doi: 10.1007/s11250-009-9368-x.

12. BiniaminT, Anwar $\mathrm{H}$. The prevalence of cystic echinococcosis in cattle slaughtered in Sebeta municipal abattoir, Central Ethiopia. Biomed J Sci Tech Res. 2018;6(1):1-5. doi: 10.26717/ BJSTR.2018.06.001285.

13. Hamidieh H, Deylami-Asl A. Specifications and fertility rate of hydatid cyst in slaughtered animal in Zanjan's Slaughterhouse in 1998. Sci J Hamadan Univ Med Sci. 1998;7:10-4. [Persian].

14. Regassa F, Molla A, Bekele J. Study on the prevalence of cystic hydatidosis and its economic significance in cattle slaughtered at Hawassa Municipal abattoir, Ethiopia. Trop Anim Health Prod. 2010;42(5):977-84. doi: 10.1007/s11250-009-9517-2.

15. Rinaldi L, Maurelli MP, Veneziano V, Capuano F, Perugini AG, Cringoli S. The role of cattle in the epidemiology of Echinococcus granulosus in an endemic area of southern Italy. Parasitol Res. 2008;103(1):175-9. doi: 10.1007/s00436-0080948-x.

16. Blancas Mosqueda M, Herrera Esparza R, Rodríguez Padilla C, Tavizón García JP, Mercado Reyes M, Badillo Almaraz V, et al. Gender as a factor of susceptibility to infection in experimental hydatidosis. Rev Latinoam Microbiol. 2007;49(1-2):31-7.
17. Hassan ZI, Mero WMS, Casulli A, Interisano M, Boufana B. Epidemiological study of cystic echinococcosis in sheep, cattle and goats in Erbil Province. Science Journal of University of Zakho. 2016;4(1):43-55. doi: 10.25271/2016.4.1.22.

18. Himonas C, Frydas S, Antoniadol-Sotiriadou K. The fertility of hydatid cysts in food animals in Greece. In: Geerts S, Kumar V, Brandt J, eds. Helminth Zoonoses. Dordrecht: Springer Netherlands; 1987. p. 12-21.

19. Ibrahim MM, Ghamdi M, Gahmdi M. Helminths community of veterinary importance of livestock in relation to some ecological and biological factors. Turkiye Parazitol Derg. 2008;32(1):42-7.

20. Al-Shaibani IRM, Saad FA, Al-Mahdi H. Cystic echinococcosis in humans and animals at Dhamar and Taiz governorates, Yemen. Int J Curr Microbiol Appl Sci. 2015;4(2):596-609.

21. McManus DP. Molecular discrimination of taeniid cestodes. Parasitol Int. 2006;55 Suppl:S31-7. doi: 10.1016/j. parint.2005.11.004.

22. Regassa A, Abunna F, Mulugeta A, Megersa B. Major metacestodes in cattle slaughtered at Wolaita Soddo Municipal abattoir, Southern Ethiopia: prevalence, cyst viability, organ distribution and socioeconomic implications. Trop Anim Health Prod. 2009;41(7):1495-502. doi: 10.1007/s11250009-9338-3.

23. Dalimi A, Motamedi G, Hosseini M, Mohammadian B, Malaki $\mathrm{H}$, Ghamari Z, et al. Echinococcosis/hydatidosis in western Iran. Vet Parasitol. 2002;105(2):161-71.

24. Daryani A, Alaei R, Arab R, Sharif M, Dehghan MH, Ziaei $\mathrm{H}$. The prevalence, intensity and viability of hydatid cysts in slaughtered animals in the Ardabil province of Northwest Iran. J Helminthol. 2007;81(1):13-7. doi: 10.1017/ s0022149x0720731x.

25. Lahmar S, Kilani M, Torgerson PR, Gemmell MA. Echinococcus granulosus larvae in the livers of sheep in Tunisia: the effects of host age. Ann Trop Med Parasitol. 1999;93(1):75-81. doi: $10.1080 / 00034989958825$. 